\title{
Proposta de uma Abordagem Computacional para Detecção Automática de Estilos de Aprendizagem Utilizando Modelos Ocultos de Markov e FSLSM
}

\author{
Edson Sena ${ }^{1}$, Alessandro Vivas ${ }^{1}$, Luciana Assis $^{1}$, Cristiano Pitangui $^{2}$ \\ ${ }^{1}$ PPGED - Universidade Federal dos Vales do Jequitinhonha e Mucuri (UFVJM) \\ Diamantina- MG - Brasil \\ ${ }^{2}$ DTECH - Universidade Federal de São João del Rei (UFSJ) \\ Ouro Branco - MG - Brasil \\ \{eds.bsena, alessandro.vivas, lupassis,pitangui.cristiano\}@gmail.com
}

\begin{abstract}
One of the current challenges is to develop computational technologies that are able to respond properly to the teaching and learning methods. For this to occur, it is essential that virtual environments provide adequate content, and are dynamic and adaptable to the needs and interests of students. This papper proposes a probabilistic approach, combines the model proposed by Felder and Silverman (FSLSM) to learning styles, with probabilistic inference techniques of Hidden Markov Models (HMM), in order to perform the inference process of the preferences of the student for a particular learning style.
\end{abstract}

Resumo. Um dos desafios atuais é desenvolver tecnologias computacionais que sejam capazes de atender corretamente aos métodos de ensino e aprendizagem. Para que isso ocorra, é fundamental que os ambientes virtuais forneçam conteúdo adequado, além de serem dinâmicos e adaptáveis às necessidades e interesses dos estudantes. Este trabalho tem como objetivo, apresentar uma abordagem probabilística, que combina o modelo proposto por Felder e Silverman (FSLSM) para estilos de aprendizagem, com as técnicas de inferência probabilística dos Modelos Ocultos de Markov (HMM), a fim de realizar o processo de inferência da preferência do aluno por um determinado estilo de aprendizagem.

\section{Introdução}

Com os constantes avanços tecnológicos, novas plataformas de apoio às atividades acadêmicas foram propostas com o intuito de atender às necessidades e particularidades individuais de cada aprendiz.

Para se adequar as particularidades dos alunos, os Ambientes Virtuais de Aprendizagem (AVA) precisam criar regras computacionais eficientes, que sejam capazes de inferir os estilos de aprendizagem a partir das ações individuais de cada estudante, durante a sua interação com o AVA (Lopes, 2009). 
V Congresso Brasileiro de Informática na Educação (CBIE 2016)

Anais do XXVII Simpósio Brasileiro de Informática na Educação (SBIE 2016)

Questionários psicométricos são métodos tradicionais para a detecção de estilos de aprendizagem. Entretanto, várias pesquisas pretendem substituir os questionários por modelos dinâmicos que inferem o estilo de aprendizagem através da utilização de técnicas de inteligência artificial.

Dentre as diversas técnicas utilizadas para o propósito, o uso de modelos ocultos de Markov (HMM - Hidden Markov Models), vem ganhando espaço nas recentes pesquisas. Esta técnica de aprendizado de máquina, que utiliza em sua estrutura modelos probabilísticos no processo de inferência, é frequentemente utilizada em pesquisas relacionadas à biotecnologia, reconhecimento de voz, previsões meteorológicas, entre outras aplicações.

O presente trabalho propõe uma abordagem computacional que utiliza a combinação do FSLSM em um modelo oculto de Markov para a detecção automática de estilos de aprendizagem. Este trabalho leva em consideração características de cada dimensão do FSLSM obtidas através da interação do estudante com o ambiente virtual de aprendizagem. Matrizes de emissão e transição de estados do modelo de Markov são criadas através do algoritmo de Viterbi.

Este artigo apresenta em sua segunda seção, os principais trabalhos relacionados ao processo de detecção e correção automática de estilos de aprendizagem em ambientes virtuais de aprendizagem, a terceira seção apresenta os principais autores e respectivos conceitos que balizam a estruturação do trabalho, a quarta seção apresenta a estrutura da abordagem proposta, a quinta seção apresenta os experimentos e resultados obtidos, finalmente na sexta seção são apresentadas as considerações finais e a proposta de trabalhos futuros.

\section{Trabalhos Relacionados}

Em modelos computacionais que utilizam variáveis não determinísticas, as redes bayesianas representam uma alternativa relevante. $O$ método tem sido utilizado na modelagem do perfil dos estudantes pelo rigor matemático e pela capacidade de lidar com as incertezas necessárias para o modelo probabilístico.

Vários autores como Piombo et al. (2003), Alkhuraiji et al (2011), fazem uso de redes bayesianas em combinação com o modelo de estilos de aprendizagem proposto por Felder e Silverman (1988). O trabalho teve como objetivo de propor um modelo computacional que pudesse ser adaptado e utilizado em ambientes virtuais de aprendizagem.

O trabalho proposto por Garcia et al. (2007), representou a base vários outros trabalhos que relacionam redes bayesianas e FSLSM. Neste trabalho os autores utilizaram o Teorema de Bayes e foram utilizados onze padrões de comportamento para enquadrar o estudante nas dimensões do modelo do FSLSM.

Özpolat e Akar (2009), propõem uma arquitetura computacional capaz de extrair e modelar os estilos de aprendizagem do estudante utilizando o FSLSM em combinação com uma estrutura de árvore de decisão que conta com o algoritmo de classificação NBTree e o classificador Binary Relevance. 
Lo et. al. (2006), propõe um ambiente virtual de aprendizagem com capacidade de adaptação aos estilos cognitivos do estudante. A aplicação é composta por um modelo do estudante e um modelo adaptativo através de Rede Neural Artificial (RNA) multicamadas para classificar o comportamento do aluno. Já Manghirmalani et al. (2011), utilizaram uma combinação de RNA (LVO - Learning Vector Quantization) e algoritmos de árvore de decisão. O modelo computacional proposto foi capaz de realizar diagnóstico e classificar de deficiências no processo de ensino e aprendizagem de escrita e cálculos matemáticos.

Dorça (2012) apresenta uma abordagem probabilística utilizando aprendizagem por reforço, onde é implementado um modelo do estudante dinâmico e interativo. $\mathrm{O}$ processo de atualização do modelo se dá a partir de informações do desempenho dos estudantes dentro do ambiente de aprendizagem.

Uma proposta para utilizar HMM no processo de detecção automática de estilos de aprendizagem em ambientes virtuais de aprendizagem é descrita em Nguyen (2014), na qual foi utilizado uma combinação dos modelos de Felder e Siverman (1988) e Honey e Munford (1992). O HMM foi construído levando em consideração as dimensões e características específicas de cada modelo para definir os estados ocultos e os observáveis. Ao final foi utilizado o algoritmo de Viterbi para realizar a inferência do estilo de aprendizagem probabilístico.

\section{Referencial Teórico}

Nesta seção, são apresentados conceitos fundamentais sobre o modelo de estilos de aprendizagem FSLSM e os Modelos Ocultos de Markov (HMM), definições essenciais para a realização deste trabalho.

\subsection{Felder-Silverman Learning Style Model (FSLSM)}

Felder e Silverman (1988), descreve os Estilos de Aprendizagem como sendo as diversas formas como os indivíduos recebem, organizam e processam as informações, com o objetivo de construção do conhecimento. O autor faz uma ressalva que essas habilidades não são estáticas, podendo ser moldadas e desenvolvidas ao longo do tempo.

Com base em outros modelos, desenvolveram um modelo estruturado que foi inicialmente direcionado aos estudantes de engenharia e composto por quatro dimensões. Percepção que se subdivide em sensitivo e indutivo. Entrada que pode ser visual e verbal. Processamento onde o estudante pode ser ativo ou reflexivo e a dimensão organização que classifica o aluno como sequencial ou global. Este modelo ficou definido com Felder-Silverman Learning Style Model (FSLSM).

Segundo Dorça (2012), o modelo de Felder e Silverman fornece dimensões dinâmicas, isto é, podem sofrer variações dentro de uma mesma dimensão, além de terem a capacidade de se adaptarem a uma outra tendência de aprendizagem. Sendo assim, o mesmo propôs a utilização de escalas ao invés tipos fixos. Isso permitiu que as dimensões do modelo fossem descritas como fortes, moderadas e leves. 
V Congresso Brasileiro de Informática na Educação (CBIE 2016)

Anais do XXVII Simpósio Brasileiro de Informática na Educação (SBIE 2016)

\subsection{Modelos Ocultos de Markov (HMM)}

De acordo com Rabiner (1989), um modelo oculto de Markov representa uma variação do formalismo clássico das cadeias de Markov, onde além das tradicionais distribuições de probabilidades, o modelo possui uma distribuição baseada na "observação e comportamento" do ambiente em que foi inserido. O modelo consiste em um processo duplamente estocástico onde um processo oculto e não observável recebe símbolos sequenciais de um outro processo que observa e monitora o comportamento do ambiente.

Em sua forma genérica, um Modelo Oculto de Markov, é formado por um conjunto de parâmetros do modelo $\lambda=(\hat{\mathrm{A}}, \mathrm{B}, \pi)$, onde $\pi$ representa $\mathrm{o}$ vetor de probabilidades inicial da cadeia de Markov oculta, Â é a matriz de transição entre os estados, já B representa a matriz de probabilidade de emissão dos estados observáveis.

Um dos problemas canônicos relacionados aos HMMs, tem como principal objetivo, encontrar a melhor sequência de estados ocultos que melhor representa o conjunto de estados observáveis do modelo. Para a solução do problema, a principal solução proposta por vários autores é o algoritmo de Viterbi que consiste em um algoritmo dinâmico e recursivo, que tem sua complexidade polinomial. (ALAMINO, 2005)

\section{Abordagem Proposta}

No contexto da organização e estrutura do FSLSM, buscou-se construir uma abordagem computacional capaz de explorar as características fundamentais das dimensões e sub dimensões do modelo.

Conforme descrito anteriormente, o FSLSM é composto pelas quatro dimensões: entrada, processamento, organização e percepção. Sendo as mesmas subdividas em entrada: visual e verbal, processamento: ativo e reflexivo, organização: sequencial e global, percepção: sensitivo e intuitivo. Nessa estrutura, cada dimensão e sub-dimensão possuem características específicas, que se apresentaram como a estrutura essencial para a construção do HMM e das suas respectivas matrizes de emissão e transição de estados.

Na dimensão entrada, observa-se que os estudantes com preferências pela subdimensão visual, tem aptidão por objetos de aprendizagem que sejam diagramas, filmes, imagens e demonstrações. Já os que preferem a sub dimensão verbal, são atraídos por objetos de aprendizagem que sejam textos, áudios e manuscritos.

Dessa forma a dimensão entrada foi organizada em um modelo oculto de Markov, onde os estados ocultos representam as duas sub dimensões, visual ou verbal. Já os estados observáveis são representados pelos objetos de aprendizagem: imagem, vídeo, texto e áudio.

Para a dimensão processamento, onde os estudantes são classificados em ativos ou reflexivos, observa-se que os estudantes com tendências para a sub dimensão ativo, tem preferência por experiências de aprendizagem em grupo, de forma coletiva, participando de chats, fóruns e pontos de encontro virtuais. Já os com tendências a subdimensão reflexivo, optam por atividades individuais, preferindo a interação com o conteúdo formal e a criação de modelos. 
V Congresso Brasileiro de Informática na Educação (CBIE 2016)

Anais do XXVII Simpósio Brasileiro de Informática na Educação (SBIE 2016)

Assim, a dimensão processamento foi descrita em um modelo oculto de Markov, onde os estados ocultos representam as duas sub-dimensões, ativo ou reflexivo. Já os estados observáveis são representados pelas preferências do estudante em se interagir em grupo participando de chats e fóruns ou priorizando as atividades individuais com o conteúdo formal e a criação de modelos próprios.

A dimensão organização, tende a observar a forma com que o estudante interage com o ambiente virtual, e pode classificá-los em sequenciais ou globais. Observa-se que os estudantes com tendências para progredirem no ambiente de forma sequencial, tem preferência por avançar linearmente no conteúdo, além de optarem geralmente por conteúdos mais analíticos. Já os com tendências à sub-dimensão global, optam por acessarem o ambiente aleatoriamente, e preferindo a interação com o conteúdo sintético, bem resumido.

Nesse contexto a dimensão organização foi construída em um modelo oculto de Markov, onde os estados ocultos representam as duas sub-dimensões, sequencial ou global. Os estados observáveis são representados pelas preferências do estudante em se interagir de forma sequencial e optando por conteúdos analíticos ou acessando os conteúdos sintéticos e de maneira global.

Na dimensão percepção, o objetivo é observar as preferências do estudante quanto ao tipo do objeto de aprendizagem, e são classificados em sensitivos ou intuitivos, os estudantes com tendências a serem sensitivos, preferem objetos relacionados às experiências reais, fatos ou dados. Já os com tendências à serem intuitivos, optam por objetos teóricos, modelos matemáticos e abstrações.

A dimensão percepção foi definida em um modelo oculto de Markov, onde os estados ocultos representam as duas sub dimensões, sensitivo ou intuitivo. Os estados observáveis são representados pelas preferências do estudante pelos objetos de aprendizagem: fatos, experiências, dados, teorias, modelagens e abstrações.

Após a definição dos estados ocultos e os estados observáveis realizou-se a organização das matrizes de transição e emissão de estados com o objetivo de estabelecer os critérios necessários para a política de transição de estados e alimentação das matrizes.

Conforme descrito anteriormente, um modelo oculto de Markov apresenta-se nas definições de Rabiner (1989) como um processo duplamente estocástico. Sendo assim o mesmo possui em sua estrutura duas matrizes de transição de estados, que armazena as probabilidades de transição entre os estados e emissão de sinais observáveis. 
V Congresso Brasileiro de Informática na Educação (CBIE 2016)

Anais do XXVII Simpósio Brasileiro de Informática na Educação (SBIE 2016)
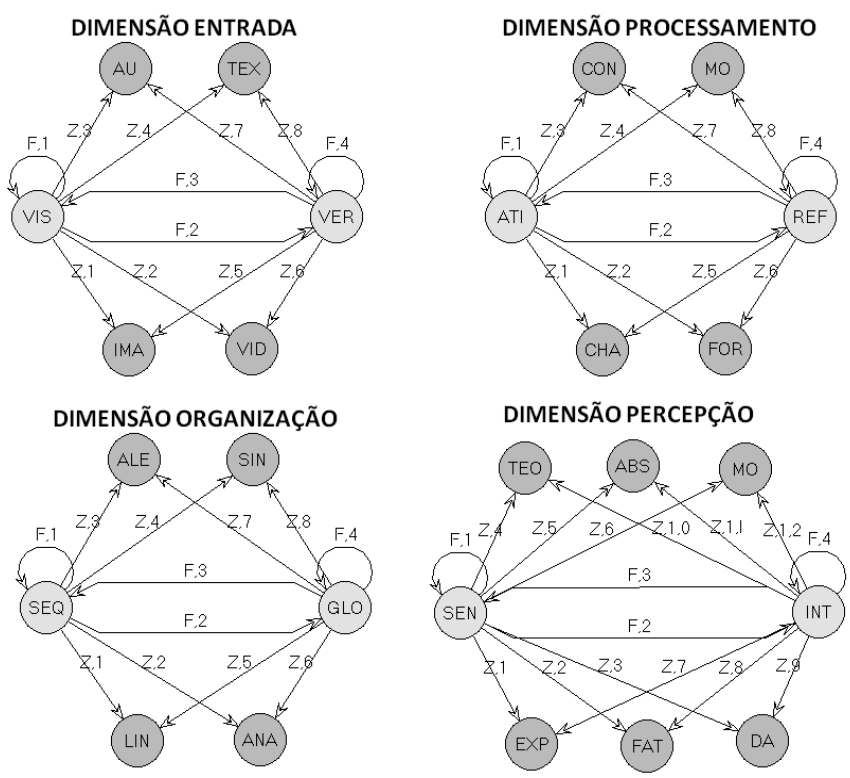

Figura 1. Modelo oculto de Markov, representando as quatro dimensões do FSLSM.

Os modelos acima apresentados, representam a estruturação das dimensões do FSLSM em quatro HMMs, demonstrando os estados observáveis e os estados ocultos do modelo, que apresentam-se capazes de gerar as matrizes de transição de estados e emissão de sinais observáveis para o modelo.

\subsection{Algoritmo de Viterbi}

Forney (1973) define o algoritmo de Viterbi como um modelo computacional recursivo e dinâmico capaz de solucionar em sua maioria, os problemas de estimativa de transição e sequência de estados em modelos ocultos de Markov de tempo discreto. Sendo o mesmo, predominante utilizado na resolução do problema de decodificação em modelos ocultos de Markov, além de ser amplamente utilizado em diversas áreas da ciência da computação.

O comportamento recursivo do algoritmo de Viterbi representa o núcleo central de sua aplicação em modelos ocultos de Markov, uma vez que é responsável pelo processo indutivo durante o cálculo e maximização da distribuição de probabilidades do modelo, o que é primordial para definição da transição entre os estados e definição da sequência de estados que melhor representa o modelo.

Já as características de programação dinâmica do algoritmo, permitem a otimização do processo decisório, etapa também fundamental no objetivo para induzir o sistema na geração da melhor sequência de estados, uma vez que permite a escolha do estado de maior probabilidade local e que posteriormente serão agrupados em um conjunto global de melhores estados e que possuem as maiores probabilidades locais.

\section{Experimentos e Resultados Obtidos}

A metodologia utilizada para análise da abordagem proposta, foi a simulação computacional, que, com base em uma revisão inicial da literatura, realizou-se a estruturação do modelo probabilístico dos estilos de aprendizagem, utilizando uma 
V Congresso Brasileiro de Informática na Educação (CBIE 2016)

Anais do XXVII Simpósio Brasileiro de Informática na Educação (SBIE 2016)

estrutura computacional que combina um modelo oculto de Markov, com as dimensões e características do modelo proposto por Felder e Silverman (1988).

Devido as características probabilísticas do modelo proposto e diversas operações com matrizes, optou-se por uma linguagem de alto nível com uma disponibilidade de recursos e técnicas estatísticas, processamento de matrizes, além da possibilidade de utilização de funções e pacotes específicos, por atender a todos estes critérios definiu-se a utilização da linguagem R Estatística, versão 3.2.0.

Não possuindo de forma nativa uma biblioteca específica para processar modelos ocultos de Markov, fez-se necessária a instalação de pacotes adicionais com este propósito, os pacotes utilizados foram: HMM, HiddenMarkov e hmm.discnp, ambos de fácil manipulação e de acesso facilitado à documentação dos mesmos.

\subsection{Processo de Simulação}

O processo de simulação buscou representar o mais próximo o comportamento do estudante perante o AVA, interagindo com os objetos de aprendizagem que lhe foram disponibilizados, para que esse processo fosse quantificado e permitindo que as matrizes necessárias para a inferência do estilo de aprendizagem probabilístico fosse o mais próximo da realidade.

O processo foi dividido em seis etapas distintas, conforme descritas a seguir.

- Pré-Processamento: transformação das características de cada dimensão do FSLSM em um número inteiro, dentro do intervalo dos pseudoaleatórios a serem gerados durante a simulação.

- Geração dos pseudoaleatórios: Utilização das funções de distribuição binomial do $\mathrm{R}$ Estatística, a fim de gerar um conjunto numérico que representasse a interação do estudante com cada característica do FSLSM. Os parâmetros de geração foram calibrados, além de uma função geradora de sementes aleatórias, com o propósito de garantir uma heterogeneidade do conjunto numérico gerado.

- Criação dos histogramas: Após o processo de criação dos números pseudoaleatórios, os dados foram quantificados e agrupados em histogramas, totalizando a quantidade de interações com cada característica definida para as dimensões e sub dimensões do FSLSM.

- Definição das matrizes de transição e emissão de estados: Nesta etapa os dados agrupados nos histogramas serviram de insumo para a definição das matrizes de transição e emissão de estados. A correta definição das matrizes constitui o elemento central para o correto processo de inferência do estilo de aprendizagem probabilístico, uma vez que ambas representam o núcleo central dos modelos ocultos de Markov.

- Inferência probabilística do estilo de aprendizagem: Nesta etapa, foi utilizado o algoritmo de Viterbi sobre o HMM gerado na etapa anterior, com o objetivo de obter a sequência de estados ocultos que melhor representa o modelo. Esta etapa, representa a ideia central da abordagem proposta, uma vez que é neste ponto que o algoritmo de Viterbi, com base nas informações da cadeia de Markov, converge para um determinado estado oculto, definindo um processo de inferência probabilística para o estilo de aprendizagem que melhor 
V Congresso Brasileiro de Informática na Educação (CBIE 2016)

Anais do XXVII Simpósio Brasileiro de Informática na Educação (SBIE 2016)

representa as características predominantes do modelo, e que foram observadas durante a interação.

- Coleta e armazenamento dos LOGs do processo: Todas as fases da simulação geraram resultados que foram armazenados em arquivos de log, através de funções nativas da linguagem $\mathrm{R}$ Estatística, que posteriormente foram importados para uma planilha eletrônica onde foram tabulados e geraram tabelas e gráficos.

\subsection{Resultados Obtidos}

A presente seção tem como objetivo apresentar os resultados obtidos durante os experimentos, aos quais foram armazenados em arquivos de log durante a execução do simulador, e posteriormente foram importados e processados em planilhas eletrônicas, gerando tabelas estruturadas e gráficos.

O FSLSM foi organizado e dividido em quatro dimensões, que definem as preferências do estudante durante as seções de aprendizagem. O processo de simulação seguiu a mesma estrutura, sendo realizado os experimentos por dimensões separadamente e apurando os resultados da mesma forma, e assim serão também apresentados. A seguir são apresentados resultados parciais dos experimentos:

Tabela 1. Mapa de apuração experimentos

\begin{tabular}{|c|c|c|c|c|c|c|c|c|c|c|c|c|}
\hline \multicolumn{13}{|c|}{ DIMENSÃO PERCEPÇÃO } \\
\hline SEM & EXP & FAT & DAD & TEO & ABS & MOD & SEN-R & INT-R & EA-R & SEN-P & INT-P & EA-P \\
\hline 1483097 & $0 \%$ & $17 \%$ & $34 \%$ & $41 \%$ & $5 \%$ & $3 \%$ & $51 \%$ & $49 \%$ & SEN & $44 \%$ & $56 \%$ & INT \\
\hline 2395547 & $24 \%$ & $33 \%$ & $32 \%$ & $10 \%$ & $1 \%$ & $0 \%$ & $89 \%$ & $11 \%$ & SEN & $97 \%$ & $3 \%$ & SEN \\
\hline 5727653 & $21 \%$ & $34 \%$ & $32 \%$ & $12 \%$ & $1 \%$ & $0 \%$ & $87 \%$ & $13 \%$ & SEN & $95 \%$ & $5 \%$ & SEN \\
\hline 9130638 & $0 \%$ & $0 \%$ & $3 \%$ & $14 \%$ & $45 \%$ & $38 \%$ & $3 \%$ & $97 \%$ & INT & $0 \%$ & $100 \%$ & INT \\
\hline \multicolumn{11}{|c|}{ DIMENSÃO PROCESSAMENTO } & & \\
\hline SEM & $\mathrm{CHA}$ & FOR & CON. & MOD & ATI-R & REF-R & EA-R & ATI-P & REF-P & EA-P & & \\
\hline 704618 & $18 \%$ & $32 \%$ & $41 \%$ & $9 \%$ & $50 \%$ & $50 \%$ & ATI-REF & $47 \%$ & $53 \%$ & REF & & \\
\hline 791402 & $1 \%$ & $14 \%$ & $42 \%$ & $43 \%$ & $15 \%$ & $85 \%$ & REF & $10 \%$ & $90 \%$ & REF & & \\
\hline 775544 & $42 \%$ & $41 \%$ & $16 \%$ & $1 \%$ & $83 \%$ & $17 \%$ & ATI & $88 \%$ & $12 \%$ & ATI & & \\
\hline 628812 & $42 \%$ & $43 \%$ & $14 \%$ & $1 \%$ & $85 \%$ & $11 \%$ & ATI & $91 \%$ & $9 \%$ & ATI & & \\
\hline \multicolumn{11}{|c|}{ DIMENSÃO ORGANIZAÇÃO } & & \\
\hline SEM & LIN. & ANA. & ALE. & SINT. & SEQ-R & GLO-R & EA-R & SEQ-P & GLO-P & EA-P & & \\
\hline 7697947 & $14 \%$ & $50 \%$ & $29 \%$ & $7 \%$ & $64 \%$ & $36 \%$ & SEQ & $86 \%$ & $14 \%$ & SEQ & & \\
\hline 5049851 & $7 \%$ & $32 \%$ & $38 \%$ & $23 \%$ & $39 \%$ & $61 \%$ & GLO & $18 \%$ & $82 \%$ & GLO & & \\
\hline 3976229 & $12 \%$ & $37 \%$ & $45 \%$ & $6 \%$ & $49 \%$ & $51 \%$ & GLO & $50 \%$ & $50 \%$ & $S-G$ & & \\
\hline 7450413 & $12 \%$ & $30 \%$ & $41 \%$ & $17 \%$ & $42 \%$ & $58 \%$ & GLO & $10 \%$ & $90 \%$ & GLO & & \\
\hline \multicolumn{11}{|c|}{ DIMENSÃO ENTRADA } & & \\
\hline SEM & IMG & VID & TEX & AUD & VIS-R & VER-R & EA-R & VIS-P & VER-P & EA-P & & \\
\hline 916514 & $24 \%$ & $37 \%$ & $32 \%$ & $7 \%$ & $61 \%$ & $39 \%$ & VIS & $92 \%$ & $8 \%$ & VIS & & \\
\hline 405842 & $8 \%$ & $41 \%$ & $44 \%$ & $7 \%$ & $49 \%$ & $51 \%$ & VER & $54 \%$ & $46 \%$ & VIS & & \\
\hline 329813 & $8 \%$ & $34 \%$ & $41 \%$ & $17 \%$ & $42 \%$ & $58 \%$ & VER & $12 \%$ & $88 \%$ & VER & & \\
\hline 496079 & $21 \%$ & $47 \%$ & $24 \%$ & $8 \%$ & $68 \%$ & $32 \%$ & VIS & $86 \%$ & $14 \%$ & VIS & & \\
\hline
\end{tabular}


V Congresso Brasileiro de Informática na Educação (CBIE 2016)

Anais do XXVII Simpósio Brasileiro de Informática na Educação (SBIE 2016)

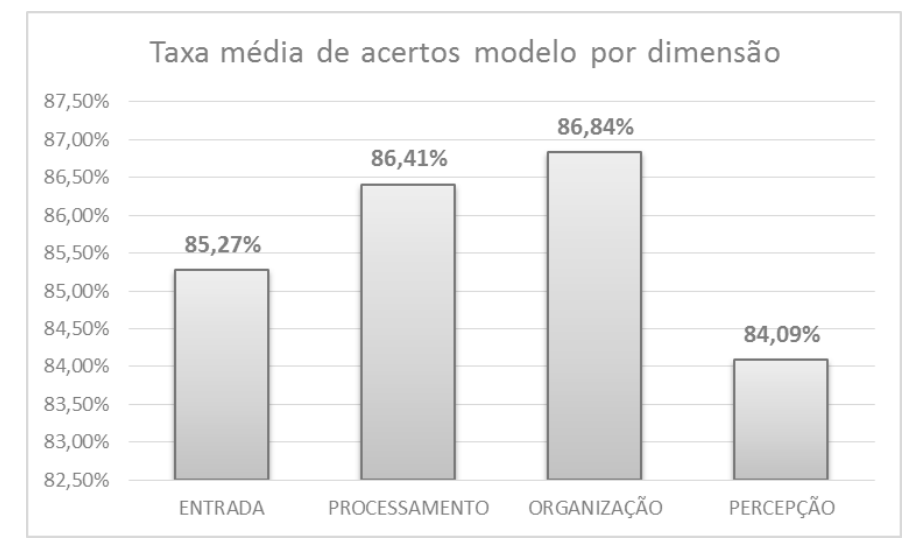

Figura 2. Gráfico apresentando a média de acertos de inferência do estilo de aprendizagem.

Avaliando de forma conjunta o desempenho da simulação nas quatro dimensões do FSLSM, percebe-se que os resultados são consideravelmente favoráveis, obtendo uma alta taxa de acerto na inferência do estilo de aprendizagem ao longo do processo de simulação. Como pode-se observar no gráfico acima.

\section{Considerações Finais}

Em consonância com pesquisas recentes relacionadas às técnicas de detecção automática de estilos de aprendizagem, que apontam o FSLSM como um dos modelos mais adequados para o processo, conseguiu-se explorar ao longo deste, sob diversas óticas, as características estruturais do modelo proposto por Felder e Silverman (1988), de forma que foi possível perceber que as quatros dimensões definidas por ele, apresentam-se amplamente capazes de identificar o comportamento do estudante ao longo das seções de aprendizagem.

Um ponto importante a ser destacado, consiste no uso de modelos ocultos de Markov em processos de inferência probabilística, que demonstrou ser amplamente eficiente quando a estrutura do modelo consegue absorver de forma clara e consistente os requisitos, especificações e características do objeto a ser modelado. Em parte, essa eficiência probabilística se deve ao fato da possibilidade de observação de informações próprias do modelo, através da influência dos sinais emitidos pelos estados observáveis no processo de transição dos estados ocultos.

Por outro lado, uma análise sobre o funcionamento do algoritmo de Viterbi, demonstrou sua importância no processo de inferência probabilística, devido à sua simplicidade de implementação, eficiência na distribuição de probabilidades, juntamente com seu baixo custo computacional, para casos em que o modelo possui um número reduzido de estados.

Os trabalhos futuros caminham em algumas direções: a adaptação da abordagem para sistemas hipermídia adaptativos para a educação, a implementação de métodos avaliativos e de correção automática dos estilos de aprendizagem, além da validação do modelo em experimentos com alunos reais em ambientes virtuais de aprendizagem.

\section{Referências}


V Congresso Brasileiro de Informática na Educação (CBIE 2016)

Anais do XXVII Simpósio Brasileiro de Informática na Educação (SBIE 2016)

ALKHURAIJI, S., CHEETHAM, B., BAMASAK, O. (2011) "Dynamic Adaptive Mechanism in Learning Management System Based on Learning Styles". 2011 IEEE 11th Int. Conf. Adv. Learn. Technol., pp. 215-217.

DORÇA, F. A. (2012) "Uma abordagem estocástica baseada em aprendizagem por reforço para modelagem automática e dinâmica de estilos de aprendizagem de estudantes em sistemas adaptativos e inteligentes para educação a distância”. Programa de Pós-Graduação em Ciência da Computação. Faculdade de Computação. Universidade Federal de Uberlândia. Uberlândia, MG.

FELDER, R. M., SILVERMAN, L. (1988) "Learning and Teaching Styles In Engineering Education". North Carolina State University.

FORNEY JR, G. D. (1973) “The viterbi algorithm”. Proceedings of the IEEE, v. 61, n. 3 , p. 268-278.

GARCÍA, P., AMANDI, A., SCHIAFFINO, S., CAMPO, M. (2007) "Evaluating Bayesian Networks precision for detecting students learning styles". Computers \& Education. Volume 49, no. 3, pp. 794-808. Elsevier. Buenos Aires.

HONEY, P.; MUMFORD, A. (1992) “The Manual of Learning styles" Peter Honey Publications; 3rd Edition.

LO, J., CHAN, Y., YEH, S. (2012) "Designing an adaptive web-based learning system based on students cognitive styles identified online". Computers \& Education, v. 58, n. 1, p. 209-222.

LOPES, R. (2009) "Planejamento instrucional adaptativo usando Workflow e planejamento genético". 2009. 140 f. Dissertação (Mestrado)-Universidade Federal de Uberlândia, Uberlândia, MG.

MANGHIRMALANI, P., ZENOBIA P., KAVITA J. (2011) "Learning disability diagnosis and classification-A soft computing approach". Information and Communication Technologies (WICT), 2011 World Congress on. IEEE.

NGUYEN, L. (2013) "A New Approach for Modeling and Discovering Learning Styles by using Hidden Markov Model". Global Journal of Human Social Science Linguistics \& Education. Volume 13 Issue 4 Version 1.0.

ÖZPOLAT, E., AKAR, G. (2009) "Automatic detection of learning styles for an elearning system”. Comput. Educ., vol. 53, no. 2, pp. 355-367.

PIOMBO, C., BATATIA, H., AYACHE, A. (2003) "A framework for adapting instruction to cognitive learning styles". IEEE.

RABINER, L. R. (1989) “A tutorial on hidden markov models and selected applications in speech recognition". Proceedings of the IEEE, 77(2):257-286. 